\title{
Chirurgische Ratschläge für den Praktiker.
}

Von G. Ledderhose in München.

XIII.

Untersuchung und Begutachtung von Unfallfolgen.

Zahlreichen Praktikern ist die Ausstellung eines Gutachtens unerwünscht, weil sie sich unsicher fühlen und weil sie sich anderseits der großen Verantwo-tung dieser Aufgabe bewtißt sind. In der Tat stellt die ärztliche Be :utachtung keine geringen Anforderungen. Der Arzt muß die gesetżichen Bestimmungen und die privatrechtlichen Verträge in ihren Beziehungen zur medizinischen Wissenschaft und Praxis im allgemeinen und bezüglich des vorliegenden Falles kennen, er muß die Untersuchungsmethoden beherrschen, welche es ermöglichen, ein genaues Bild von dem allgemeinen Gesundheitszustand des Untersuchten und von den besonderen, bei ihm bestehenden krankhaften oder traumatischen Veränderungen zul gewinnen, er muß ferner imstande sein, daraus die richtigen Schlüsse zu ziehen und sie in überzeilgender Form zu Papier zu bringen. Sehr einfachen, in kurzer Zeit zu erledigenden Gutachten stehen andere gegenüber, die zeitratubende Vertiefung in den Gegenstand und literarisches Studium erforderlich machen. Nur durch Erfahrung und Uebung läß sich die nötige Sicherlıeit erwerben.

Der Untersuchung des Kranken hat wie üblich dessen Bericht über Ursache und Verlauff seines Leidens sowie über seine Beschwerden und Klagen vorauszugehen. Dabei ist scharf im Auge zit behalten, daß namentlich in allen Fällen, wo das ärztliche Gutachten in Beziehung steht zu wirtschaftlichen und materiellen Interessen, geprïft werden muß, ob nicht bewußt oder lunbewußt falsche Angaben gemacht werden und ob die entgegengenommenen Berichte und Klagen nicht durch fremde und eigene Suggestion wesentlich beeinflußt sind. So wünschenswert es ist, den Kranken ausreden zu lassen und seine Darstellung möglichst wenig durch Einwände und Fragen zu unterbrechen, so unvermeidlich ist es, sich durch Befragen Aus-

1) Zschr. f. Krebsforsch. 1913, 13, S. 1. - 2) Erscheint ausfïhrlich mit Literaturangaben demnächst in den ${ }_{n}$ Fortschritten der Medizin". 
kunft zu verschaffeı über Dinge, die den ärztlichen Sachverständigen vorwiegend interessieren. Immer mehr hat es sich gezeigt, wie schädlich sog. Suggestivfragen von seiten des Arztes wirken. Man soll es also vermeiden, den Kranken z. B. zul fragen, ob er diese oder jene Klagen hat, ob diese oder jene betastete Stelle schmerzt. Der untersuchende Arzt bedarf weitgehenden psychologischen Verständnisses, um alle ihm gemachten Angaben richtig bewerten zu könnell.

Die Regel, daß bei jeder eingehenden ärztlichen Krankenuntersuchung neben der Befundaufnahme an den erkrankten oder verletzten Körperteilen ein Urteil über den Ernährungs-und al1gemeinen Gesundheitszustand sowie über die Funktion der wichtigsten Organe gewonnen werden muß, gilt auch für die Vorbereitung auszustellender Gutachten. Ein Mißverhältnis zwischen Körpergröße und Körpergewicht sowie krankhafter Allgemeinzustand können auf Unterernährung oder auf Organveränderungen schließen lassen, die vielleicht in ursächlichem Zusammenhang mit erlittenen Unfällen stehen. Die Untersuchung des Herzens, des Pulses, des Blıtdrucks, der Lungen, des Urins, ferner die Betastung der Bauchorgane sowie die Prüfung der Pupillenreaktion und der Sehnenreflexe, endlich insbesondere bei älteren Personen die Feststellung der Gelenkbeweglichkeit ergibt die Unterlagen für den Allgemeinbefund. Aus dem großell Aufgabenkreis der ärztlichen Begutachtung seien für die naclsstehende Betrachtung die Folgen einiger besonders wichtiger Unfallverletzungen an den einzelnen Körpergegende ${ }^{1}{ }^{1}$ ) herausgegriffen.

Bei der Nachuntersuclumg der Kopfverletzten lassen sich sehr häufig keine bestimmten objektiven Unfallfolgen zur Erklärung der Klagen über Kopfschmerzen, Schwindelgefühil nnd Gedächtnisschwäche feststellen. Diese Fälle bildeten früher die Hauptvertreter der sog. traumatischen Neurose. Die erweiterten neueren Erfahrungen haben gezeigt, daß dieses Krankheitsbild zu Unrecht aufgestellt war und daß die genannten Klagen vorausgesetzt, daß nicht nachweislich eine Verletzung des Gehirns stattgefunden hatte, und namentlich dann, wenn sie nicht bald nach dem Unfall, sondern erst Woclıen und Monate später vorgebracht wurden, überwiegend häıffig unter den Begriff der Begehrungsvorstellungen fallen, die an sich keinen. Anspruch auf Rentenbewilligung begründen und fast sicher nach Gewährung einer Abfindung verstummen. Auch hat sich gezeigt, daß der obiektive Wert zahlreicher sog. nervöser Erscheinungen, denen man früher große diagnostische Bedeutung beimaß, nur gering ist, daß vielmehr veränderte Stimmung, Druck- und Klopfempfindlichkeit, Pupillendifferenz, Fehlen des Augapfel- und Gaumenreflexes, Einschränkung des Gesichtsfeldes, Zittern der Zunge und der Gliedmaßen, Beschleunigung des Pulses, Nachröten der Haut, Steigerung der Sehnenreflexe und Störungen der Sensibilität sehr häufig durch eigene und fremde (Aerzte) Suggestion bedingt sind und daß sie nur unter besonderen Umständen als eigentlich krankhaft angesehen werden dürfen.

Eroibt die Anamnese, daß etwa in unmittelbarem Anschluß an den Unfall die Zeichen der Basisfraktur aufgetreten waren, oder daß ausgesprochene Störung des Bewußtseins als Ausdruck der Bemit der Möglichkeit oder Wahrscheiulichkeit zu rechnen haben, daß sich nachträglich noch Beschwerden geltend machen, während bei Verletzungen der Schädelweichteile (abgeseheı von dünnen, breit mit dem Knochen verwachsenen Narben) sowie bei einfachen Schädeldachbrüchen mit folgenloser Heilung gerechutet werden kanı. Diese Erfahrungen lassen sich für die Beurteilung der Glaubwürdigkeit vorgebrachter Klagen verwerten. Kopfschmerzen sind ein so weitverbreitetes Krankheitssvmptom, daß in jedem Fall, wo nach Kopfverletzung darïber geklagt wird, festzustellen ist, ob nicht eine anderveitige Ursache vorliegt wobei die Untersıchung der Augen, der Olıren, der Nase, des Blutdrucks und des Urins vorwiegend in Betracht kommt. Große Schwicrigkeiten können sich ergeben, wenn zu entscheiden ist, ob Klagen über $S \mathrm{chwindelgefühl}$ zunächst einmal berechtigt sind und ob sic beiahendenfalls mit vorausgegangener Konfverletzung in ursächliclienı Zusammenhang stelıer. Namentlich handelt es sich darum, festzustelle $u$, ob Verletzungsfolge oder Erkrankung des Labyrintlis vorliegt. Werden epileptische Anfäll e in Beziehung zu Kopfverletzungen gebracht, so ist cin wissenschaftlich begriindetes Urteil fast trie ohne längere Krankenhantsbeobaclıtıng möglich. Ob bei Gelegetuheit von Unfällen, die deı Kopf betrafen, Verletzung des Geliirns stattgefuntlen hatte und ob Folgen zurückgeblieben wareı, mıß sich aus der Prüfung des psychischen Verhaltens und der organischen Funktionen des Gehirns ergeben.

Für die Beurteilung von Uufallfolgen des $\mathrm{Halses}$ ist im Auge zu belıalten, daß Verletzungen der Halswirbelsäule außer durch Sturz kopfüber auch durch direkte Gewalteinwirkung gegen den Nacken, durch Fall auf die Füße, ja selbst durch Muskelzıng zustandekommen und daß die Röntgentuntersuclıung eine weit größere Häufigkeit de $\mathrm{Halsw}$ irbelbrüche, in Gegensatz zu deı einfachen Distorsionen, nachgewiesen hat, als man frülıcr angenommen hattc. Zuweilen handelt es siclı um die Untersclicidung von rein traumatischen Veränderungen und von tuberkulöser Spondvlitis.

Unzweifelhaft wird die Anfangsdiagnose Rippenbruch viel öfter gestellt, ils es berechtigt ist, was die Verletzten leicht dazu verleitet, sich für mehr geschädigt $z u$ halten, als sie es tatsächlich sind. Mau darf damit rechnen, daß namentlich Brüche mehrerer

Vgl. Ledderhose, Spattfolgen der Unfallverletzungen, ihre Untersuchung und Begutachtung, Stuttgart, F. Enke, 1921
Rippen lokale Folgen hinterlassen, die bei sorgfältiger Untersuchung nicht übersehen werden. Oefter wird mit Unrecht Interkostalneuralgie als Folge von Brustverletzung angenommen, weun die bezeichnenden Druckpunkte (nahe an der Wirbelsäule, nahe am Brustbein und in der Mitte des Nervenverlaufs) feltlen. Hatte AnspieBung der Lunge etwa durch ein Rippenbraclistück oder Zerreißung der Luıge stattgefunden (sofortiger Blutauswurf), so können Verwachsungen der Pleurablätter und Schwartenbildung zurückbleiben, was anßer durch Perkussion und Anskultation mittels der Rönttgenstrahlen festzustellen ist. Hemmintg der Atmung und namentlich nach anstrengendem Arbeiten auftretende Beschwerden sind zuweilen gegeben.

Steht der ursächliche Zusammenhang zwischen Brustverletzung und Lungentuberkulose in Frage, so ist es bereclitigt, sich an die Erfahrungstatsaclie zu halten, daß ein solcher Zusammenhang in bestimmter Form sehr selten ist. „Es muß eine der Gewißheit nahekommende Häufung pon Wahrscheinlichkeitsgründen dafür vorliegen, daß der Unfall von wesentlichem Einfluß auf die Ausbreitung oder die Entwicklung des Leidens gewesen ist, derart, daß ohne das Dazwischentreten des Unfalles der Verlauf sich wesentlich anders gestaltet haben würde, als es tatsäcllich der Fall gewesen ist." uber den Gesundheitszustand des Kranken vor dem Unfall einzuziehen, die Einzelheiten des Unfallereignisses festzustellen, das Verhalten des Kranken unmittelbar nach dem Trauma zu erfahren (Shokersclieinungetı, Klagen, Aussetzen der Arbeit) sowie Art und Menge des etwa in den ersten 48 Stunden ausgehustetell Blutes zu kennen. Jm ein möglichst genautes Urteil über die Lokalisation und die Schwere der bestehendeı tuberkulösen Lungenerkrankung zu gewinnen, müssen die verschiedlenartigen physikalischen Untersuchungsmethoden neben dem bakteriologischen Befund und die Krankenhausbeobachtung herangezogen werden.

Nicht gerade häufig werden Unfallverletzungen für die Entstehung oder Versclitimmerung von Erkrankungen der Ba uchorgane verautwortlich gemacht. Es sind zu neunen: Magengeschwüre, Appendizitis, Nierenleiden, Wanderniere, Gebärmuttervorfall. Nur wenige Aerzte sind in der Lage, auf Grund eigener Erfahrungen allein ein maßgebendes, wissenschaftlich begründetes Gutachten in derartigen Fällen zu erstatten: fast stets muß vielmehr neben gründlichem Aktenstudium und eingehender, wiederholter Untersuchung des Kranken die einschlägige Literatur zu Rate gezogen werden.

Bezüglich der wichtigen Frage, inwieweit $\mathrm{E}$ ing e we id e brü che als Unfallfolge angesehen werden dürfen, haben sich im Laufe der Zeit bestimmte Grundsätze herausgebildet, welche den betreffenden Begutachtungen als Richtschnur dienen sollen. Entscheidend ist die Erfahrungstatsache, daß fertige, aus Bruchsack mit Inhalt bestehende Hernien, insbesondere ä ußere Leis tenhernien, durch einmalige Ueberanstrengung nur dann erstmalig entstehen, weın ein präformierter, leerer Bruchsack vorher vorhanden war. Hat sich infolge von Entwicklumgshemmung (Offenbleiben des Processus vaginalis peritonei) oder von selbstä̈ndigem Wachstum einer leeren Bruchanlage oder von "Minierarbeit" eines in eine solche vorgelagerten Netzzinfels ein richtige: Bruclisack gebildet, so ist dessen plötzliche, erstmalige traumatische Füllıng überwiegend häufig von heftigen Sclımerzen, Brechreiz, Erbrechen, Shokerscheinungell und Ohnmacht begleitet, die Arbeit wird sofort niedergelegt, und es wird ärztliche Hilfe in Anspruch genomment. Die gleichent Folgen köumen auftreten, sobald ein bereits vorhandener Bruch plötzlich gewaltsam wesentlich stärker gefüllt wird und dadurch eine bleibende, wesentliche Vergrößerung erfährt. Ausnahmsweise besteht Anlaß zur Annahme erstmaliger, unfallweise Umbildung einer Bruchanlage oder eines leeren Bruchsacks zur Hernie, obwohl ernstere Erscheinungen, wie sie oben aufgezählt sind, nich aufgetreten waren und dem Kranken das Vortreten der Eingeweide nur als yoriubergehencle Spanmungs- und Schmerzempfindung zum Bewußtsein kam, wovon er seiner Umgebung Mitteilung machte.

Da die überwiegende Mehrzahl der inmeren, direkten Le iste nbrüche bei illteren Personen olute Vernittlung angeborener Anlage auf Grund yon Erschlaffuur und Atronhie der Bauchmuskeln und Spaltbildung in der Gegend tles medialen Leistenringes entsteht, kommt das Trauma in Form der Ueberaustrengung nur äıßerst selten als ursächliches Moment in Betracht, und daun sincl vom unfallchirurgischen Standnumkt besouders strenge Auforderuigen zu stellen. B ruch. a nlage und weiche Leisten (meist doppelseitig weiter äußerer Leistenring bzw. Nachgiebigkeit der vorderen Bauchwand in der Gegend des Leistenkanals sowie deutlicher Anprall der Eingeweide gegen den eingeführten Zeigefinger beim Husten und Pressen) haben neist keine Neigung zu weiterer Steigerung im Sinne der Bruchbildung und entsteheut nicht infolge von Unfallereignissen.

Die Untersuchung auf Leistenbruch hat am stehenden und liegenden Kranken zu geschehen. Zuerst wird der Samenstrang abgetastet und festgestellt, ob ein Gewebsstrang neben ihm durch den Leistenring in den Hodeusack herabsteigt, was ohne weiteres für Hernie spricht Bei freiem Samenstrang dagegen gehört eine bruchähnliche, im Hodensack liegende Geschwulst dem Hoden an Falls eine Hernie besteht, ist deren Lage, Gestalt, Inhalt, ferner die Richtung ihres Verlaufs, ihre Spammung, ihre Reponibilität sowie die Weite des äußeren Leistenriugs und des Leistenkanals festzıtstellen. Kleine Leistenbrüche weichen dem untersuchenden, mit der vorgestülpten Skrotalhaut bedeckten Finger leicht aus. Um sie naclizuweisen, muß der Finger im Kreis um ihre Basis geführt werden, ohue einen seitlichef Druck auszüuben. Stets sind beide Seiten miteinander 7I vergleichen. Bei völlig übereinstimmenden Verhältnissen beiderseit 
ist einseitige traumatische Bruchentstehung sehr unwahrscheinlich. Findet alsbald nach einem angeblichen Unfallereignis die Untersuchung statt und wird ein etwa hühnereigroßer Bruch mit weiter Pforte gefunden, dessen Inhalt leicht, z. B. bereits in der Rückenlage, ohne wesentlichlen äıßeren Druck in die Bauchhöhle zurücktritt, so beweist dies bestimmt ein älteres Bruchleiden.

Die anatomischien und mechanischen Eigenschaften des $\mathrm{Sch}$ en. kelbruches sprechen von vornherein entschieden gegen die Möglichkeit unfallweiser Entstehung; eine solche konumt denn auch nur ganz ausnahmsweise ernstlich in Frage. Die früheren Anschauungen über die Häufigkeit des traumatischen Ursnrungs von epigastrischen Hernien sowie auch über ihre klinisclie Bedeutung haben sich in neuerer Zeit sehr wesentlich geändert. Es ist der Ansicht Melchiors zuzustimmen, daß es sich in der Mehrzahl der Fälle um harmlose Fettgewebsbrïche und nicht um Bruchsäcke mit Netzinhalt handelt und daß vielleicht nur in 10\% der Fälle namhafte Beschw'erden bestehen. Sehr häufig begegnet man den verschiedenten Formen der epigastrischen Hernie als zufälligem Nebenbefund, ohne daß die Betreffenden sich ihres Leidens bewußt sind. Wenn unter diesen Umständen ein Unfallereignis platzgreift, so ereignet es sich wohl, daf die Aufnerksankeit zum erstenmal auf den schon lange vorhandenen Bruch gelenkt und er dann irrigerweise als Verletzungsfolge angesehen wird. Die traumatische Entstehung der epigastrischen Hernie kann nur dann in Betracht gezogen werden, welln der Unfall unzweifelhaft feststeht, sowie wetn erhebliche Reizerscheinungen unmittelbar nach ihm auftraten und ärztlich beobachtet wurden.

Aus der Anerkennung irgendeiner Form von Eingeweidebruch als Unfallfolge ergibt sich das Anrecht auf Bewilligung einer R e n t e nentschädigung von etwa $100 \%$.

(Schluß folgt.) 